\title{
SEBORRHOEIC DERMATITIS
}

\author{
By I. R. MARRE, M.R.C.S., I.R.C.P. \\ (Skin Physician to the Acton and Evelina Hospitals)
}

Seborrhoeic dermatitis is an acute or chronic inflammatory condition of the skin, occurring mainly in certain areas of the body, but able to spread to any part. It is commonly associated with a scaly eruption of the scalp.

\section{Etiology}

Malassez in 1874 described the Pityrosporon, a yeast-like fungus (the 'Flask' bacillus), found constantly in pityriasis capitis and seborrhoeic dermatitis, but not in other scaly lesions of the body. It is thought by many to be the cause of the condition; by some to be a saprophyte.

There are other factors which have an undoubted etiological significance. Some people have a type of skin associated with an increased output of sebum or grease (seborrhoea oleosa). The skin is muddy and somewhat thickened, with enlarged pilosebaceous follicles, and is particularly liable to 'seborrhoeic' diseases, such as pityriasis capitis, seborrhoeic dermatitis and acne. The condition is functional and often familial, and affects particularly the forehead, nose, sternal and interscapular regions, groins and genital organs.

An endocrine disturbance or imbalance is strongly suggested by the fact that seborrhoea and its associated complications often start about puberty. A dietary factor is sometimes present, as certain foods undoubtedly act as a contributing factor.

Macleod and Muende define the nature of seborrhoeic dermatitis: 'Seborrhoeic dermatitis is probably due to an infection with a yeast-like fungus, the pityrosporon, which finds a suitable soil for its growth in a skin naturally greasy from an excessive sebaceous flux.'

\section{Description}

On the scalp this may consist in the mild form of a powdery scaly condition known as dandruff. Other cases may show a yellowish, greasy scaliness on an erythematous base, which may develop into oozing crusting lesions, diffuse or in patches. There is a varying amount of itching, sometimes severe, and the condition is often associated with the loss of hair, which in the affected areas becomes frayed and brittle, and tends to fall out. This hair loss is particularly found about the temples and forehead.

Accompanying the acute condition of the scalp, the ears are often affected. Redness, scaliness, fissuring, oozing and crusting occur, especially in the post-auricular region, and may spread down the neck. The scalp eruption may extend below the hair margin in a sharply defined inflammatory line covered with crusts, known as the Corona Seborrhoica: The condition is contagious and spreads by scales shed from the scalp on to the glabrous skin. It may also be spread by infected combs and brushes. The disease may remain confined to the scalp for months or years, or may spread rapidly to the adjacent areas, or to other parts of the body.

On other parts of the body the condition first appears as round or oval scaly macules or papules, pinkish-yellow in colour, and developing into two main types of lesions : (I) a scaly erythematous type, which affects the seborrhoeic areas especially ; (2) an oozing fissured type which occurs in the flexural regions.

On the face the first type is more common, with greasy scales on an erythematous base, affecting the cheeks and forehead. The condition tends to become moist, oozing and crusting about the nose and behind the ears. 


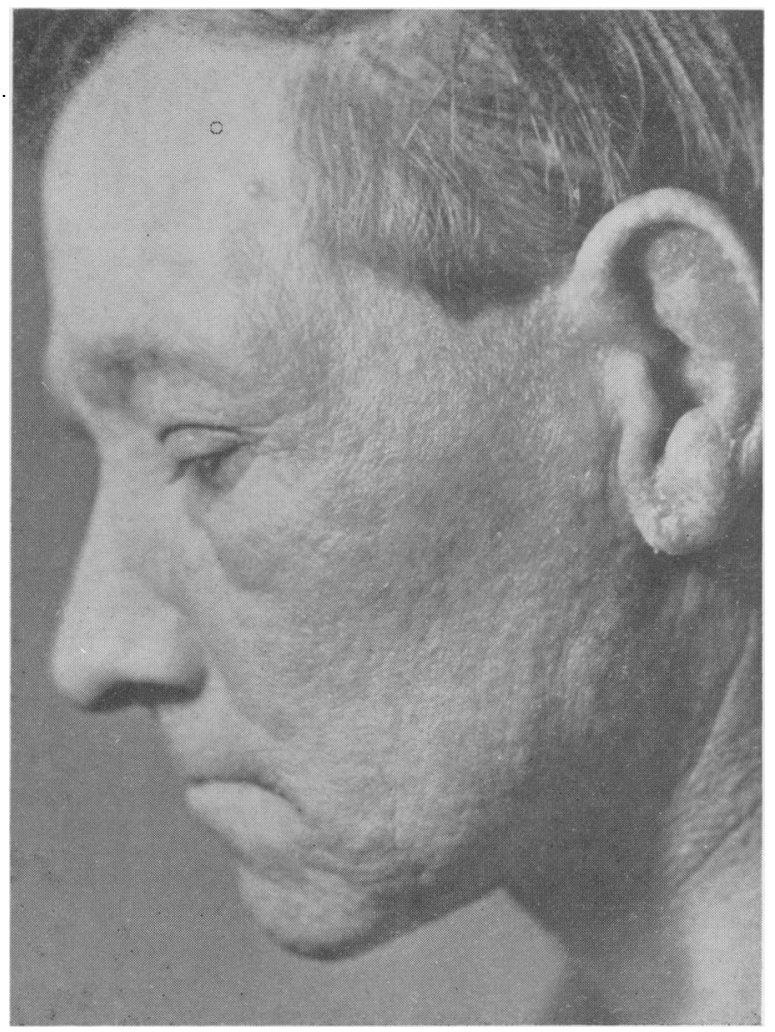

(Photograph by Dr. L. B. Bourne)

Acute seborrhoeic dermatitis of the ear, together with characteristic enlarged pilosebaceous follicles of the face. 


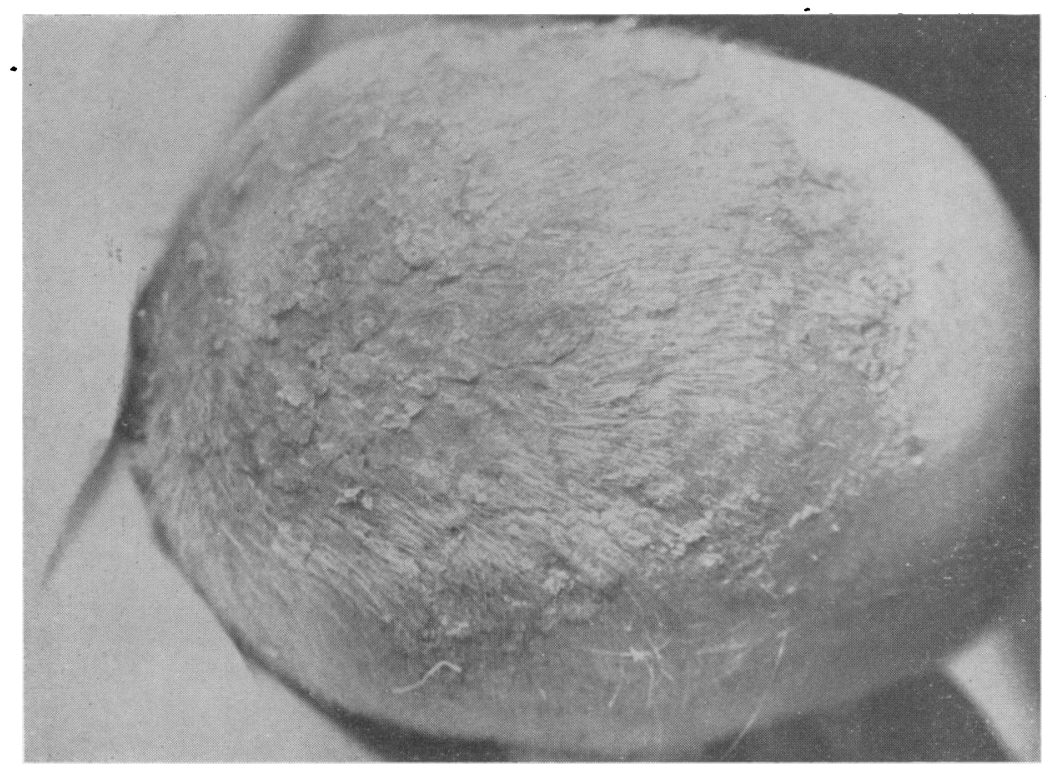

(Photograph by Dr. L. B. Bourne)

Crusted seborrhoeic dermatitis of the scalp. 
A reddened scaly state of eyebrows and evelid margins is common.

On the trunk the frequent situations are the sternal and interscapular areas, where is found the 'characteristic 'flannel-rash' or petaloid type of eruption with polycyclic gyrate figures, of a brownish-yellow colour, enlarging peripherally. The centre tends to become clear, but sometimes remains scaly, and the spreading reddish border may be covered with scaly papules. Greasy, scaly, yellowish patches on an inflamed base also occur, and it is common to find papular and macular satellite lesions surrounding the larger areas.

In the flexures the condition usually becomes moist and oozing and often spreads rapidly to the adjacent areas. Trauma and rubbing may convert the condition into an acute eczematous one, or even to an exfoliative dermatitis.

On the limbs the flexures are also commonly affected, and here the rubbing of clothes, associated with constant movement, often leads to a thickened lichenified condition of the skin. The flexor aspects may show follicular patches.

\section{Diagnosis}

On the scalp pityriasis has to be distinguished from psoriasis, in which the lesions are more circumscribed, the scales are not greasy, and typical patches may be found elsewhere on the body. Psoriatic scales do not show the pityrosporon. In children ringworm of the scalp gives partly bald patches with typical hair stumps, in which the fungus can be demonstrated.

On the face it may be necessary to differentiate Lupus erythematosus. This affects chiefly the central butterfly area of the face; the scales are dry and tenacious, and the typical plugging of the enlarged follicules are diagnostic. Impetigo pityrodes or furfuracea occurs in dry circinate patches on the face, is not inflammatory and does not usually occur near the scalp.

On the trunk psoriasis has dry and silvery scales which, when scraped off, leave the characteristic velvety oozing surface. Psoriasis tends to affect the extensor surfaces ; seborrhoeic dermatitis, the flexor.
In Pityriasis Rosea, pink oval plaques in the distribution of the rib lines are found, often with a herald patch. The essential lesion shows a pink scaly border, with a fawncoloured crinkled centre, and the disease runs an acute course of about six weeks. Ringworm patches are usually well defined, with a clearing centre and a vesicular or scaly edge, in which the fungus is found on microscopical examination. Pityriasis Versicolor is a fine scaly condition of café-au-lait macules or patches on the trunk, caused by the Microsporon furfur, which can be recognized microscopically. Erythrasma, a rare condition, caused by a fungus, the Microsporon minutissimum, gives rise to reddish or brownish plaques in moist areas, with no central clearing. Other forms of dermatitis do not show pityriasis capitis, or the greasy circumscribed lesions, with a tendency to spread peripherally.

\section{Treatment}

It is wise in every case of seborrhoeic dermatitis to treat the scalp thoroughly and systematically. In mild cases it may be sufficient to rub in the following lotion once or twice daily :

B Acidi Salicylici ........... gr. 20

Hydrargyri Perchloridi ........ gr. $\frac{1}{2}$

Liq. Carbonis deterg. . ..... m. I5

Olei ricini $\quad \ldots \ldots \ldots \ldots \ldots$ m. ro

Spirit vini rect. or spirit meth.

indust. $\quad \ldots \ldots \ldots \ldots \ldots \ldots,{ }^{5} 3$

Aq. ad. $\ldots \ldots \ldots \ldots \ldots \ldots \ldots, \quad \xi^{3}$

and shampoo the hair out once or twice weekly with Spirit Sqap

$\mathrm{R}_{x}$ Saponis viridis

Spiritus meth. industrialis. . . . $\overline{\mathbf{a}} . \overline{\mathbf{a}}$. p. aeq.

In the more greasy and crusted lesions of the scalp, the following ointment may be used, and should be persevered with even in the eczematous type :

R Sulphuris praecipitati ....... gr. Io

Acidi Salicylici ........... gr. ro

Adeps Benzoinat $\ldots \ldots \ldots \ldots$ ad. $I$

Resorcin may be substituted for the precipitated sulphur, but darkens fair hair. This should be massaged well in twice daily and shampooed out once or twice weekly. Later, when the scalp is drier, the lotion may be substituted and persisted with for some time.

In the frankly weeping eczematous condition 
of the scalp, the hair should be clipped, and the moist areas treated with very frequent applications of lead lotion (made by adding I drachm of liquor plumbi subacetat. fort. to a pint of cold tap water) for a day or two. When dry, the ointment should be substituted.

On the glabrous skin the following lotion is suitable for mild conditions :

R Sulphuris praecipitati ....... gr. 5

Tr. quillaia $\ldots \ldots \ldots \ldots \ldots$ m. Io

Lotio calaminae B.P.C. ..... ad. そ I

More severe conditions will respond to :

$\mathrm{R}$ Sulphuris praecipitati ....... gr. Io Acidi salicylici ........... gr. Io

Unguentum aquae rosae ...... ad ${ }^{\prime} \mathrm{I}$

or the following ointment :

R Hydrargyri ammoniati ....... gr. Io

Acidi salicylici ........... gr. ro

Zinci oxidi ............. 5

Paraff. molle album ........ ad $\bar{z}$ I

In the acute, weeping, flexural type moist dressings or baths are indicated. Baths of I $: 8,000$ or I : I0,000 potassium permanganate twicedaily, or continuous wet dressings -of lead lotion, may be ordered. Alternatively, $\frac{1}{2}$ per cent. silver nitrate in distilled water applied twice daily is more than useful. When dry, these areas may be treated with the calamine and sulphur lotion. Greasy ointments are borne badly in these regions, but if the calamine lotion prove too drying the following liniments may be used :

R Sulphuris praecipitati ....... gr. 5

Calaminae prep. .......... gr. 30

Zinci oxidi ............ gr. 20

Liquor calcis $\ldots \ldots \ldots \ldots \ldots . . \ldots 2$

Olei olivae or arachis......... ad. $\xi_{1}$
R Sulphuris praecipitati ........ gr. 5 Lotio calaminae oleosa (N.W.F.) ad. $\xi^{2}$

Ichthyol may be substituted for the sulphur.

For the seborrhoeic blepharitis the two per cent. yellow oxide of mercury ointment applied twice daily clears up the eruption.

\section{General Treatment}

When mild, seborrhoeic dermatitis responds readily to local treatment, but there is a tendency to recur especially in the scalp. More severe conditions may prove difficult and indeed almost intractable, and general measures should be instituted.

A holiday with plenty of fresh air should be advised, or general irradiation with ultraviolet light. The diet should be restricted in carbohydrates, and often small doses of thyroid (Tabs. thyroid. sicc. gr. $\frac{1}{2}$ b.d.). are useful. Sutton and Sutton ${ }^{2}$ advise a low fat diet.

Clothing should be light and warm and wool should not be worn next to the skin. Under conditions of increased heat and moistures relapses are common, and the condition is more difficult to cure in the tropics.

Occasionally chronic and refractory cases are improved by shock therapy with nonspecific proteins, or with autohaemotherapy. $\mathrm{X}$-ray in fractional doses on resistant patches is useful, but in view of the tendency of the disease to recur, it should not be used in treating repeated attacks in the same area.

REFERENGES

I. MACLEOD, J. M. H. and MUENDE, I. (I940), Pathology of the Skin, 83 .

2. SUTTON, R. L. and SUTTON, R. L., Jun. (4th Fd., I941) Introduction to Dermatology, 248, 6 I 5 .

or

A Clinic for the diagnosis and treatment of Internal Diseases (except Mental or Infectious Diseases). The Clinic is provided with a staff of doctors, technicians and nurses.

The surroundings are beautiful. The climate is mild. There is central heating throughout. The annual rainfall is 30.5 inches, that is, less than the average for England.

The Fees are inclusive and vary according to the room occupied.

For particulars apply to THE SECRETARY, Ruthin Castle, North Wales.

Tolegrams: Castle, Ruthin.

Telephone: Ruthin 66. 
to rest in the lukewarm bath for 20 minutes and to dry in the air. Later both epidermophyta and trichophyta respond well to Whitfield's ointment or Castellani's paint, and the monilia to I per cent. gentian violet. The greatest care to prevent re-infection must be taken. Cotton socks should be worn beneath ordinary socks or stockings, and boiled and changed daily. Hyperkeratotic areas may be treated with 6 per cent. salicylic acid ointment, until they are flat, and later with the usual fungicides.

Footwear may be sterilized by shutting in an air-tight box with formaldehyde, so that the vapour is allowed to act for four or five days.

\section{The Nails}

Ringworm of the nails, or onychomycosis, may begin independently, but often occurs with fungus infection elsewhere on the body. Infection usually takes place at the free edge of the nail, when the nail bed is attacked, and a dirty grey scaly thickening raises the nail from its bed. It spreads down the nail and grows up into the nail plate, which becomes discoloured and thickened, and finally crumbles. Infection may, however, pass under the lateral border of the nail, first attacking one side with ridging of the nail, but gradually passing across and affecting the whole nail. The infection is due to trichophyta or epidermophyta.

Diagnosis. Psoriasis of the nails may resemble fungus closely, but is not so irregular, and no fungus can be demonstrated. Syphilis of the nails is usually associated with signs elsewhere.

Treatment. Fungus infection of the nails is difficult to cure and demands unremitting attention on the part of the patient. In early infection, with only part of the nail affected, this part should be scraped away with a piece of broken glass or sharp knife until there is no obvious infection. This should be done daily, and Whitfield's ointment is applied under strapping each night. Persistence with this treatment, for months if necessary, will result in a cure.

If much of the nail is affected, surgical evulsion should be performed, and the thickened nail bed scraped. After a few days, continuous application of Whitfield's ointment should be insisted on until the nail is fully grown.

Ringworm fungi of the body, and particularly of the feet, are capable of sensitizing a patient, with the production of an allergic or sensitization eruption, which is itself free from fungus. A lichenified dermatophytide (or mycide) has been described on the trunk due to tinea capitis, but more characteristic in ringworm infection is the 'pompholyx' type of eruption on the hands. This consists of tiny vesicles along the sides of the hand, on the palms and between the fingers, and these vesicles contain no fungus. Treatment of the primary focus results in the spontaneous clearing up of the secondary sensitization eruption.

\section{CORRECTION}

Vol. XXIII, No. 257. March 1947, page 165.

SEBORRHOEIC DERMATITIS :-

Treatment.

The first formula given should be corrected to read :-

R Acidi Salicylici .......... gr. 20

Hydrargyri Perchloridi ..... gr. $\frac{1}{2}$

Liq. Carbonis deterg. .... m. I5

Olei ricini ........... m. ro

Spirit vini rect. or spirit meth.

indust. $\ldots \ldots \ldots \ldots \ldots$ ad. $\overline{3} \mathrm{I}$ 